Artículo científico

Volumen 31(1):31-42. Enero-abril, 2020

e-ISSN 2215-3608, doi:10.15517/am.v31i1.37178

http://www.revistas.ucr.ac.cr/index.php/agromeso

\title{
Interacción genotipo por ambiente de nueve variedades de algodón para los Valles interandinos en Colombia ${ }^{1}$
}

\section{Genotype by environment interaction of nine cotton varieties for inter Andean Valleys in Colombia}

\author{
Julian Roberto Mejía-Salazar', Carlos Hernando Galeano-Mendoza ${ }^{3}$,Esteban Burbano-Erazo ${ }^{4}$, \\ Franco Alirio Vallejo-Cabrera ${ }^{5}$, Miguel Arango ${ }^{6}$
}

1 Recepción: 7 de mayo, 2019. Aceptación: 9 de setiembre, 2019. Este trabajo formó parte de la tesis de maestría del primer autor, titulada "Evaluación de la interacción genotipo por ambiente para variedades transgénicas de algodón Gossypium hirsutum L", la cual se encuentra en el repositorio de la Universidad Nacional de Colombia, sede Palmira, Valle del Cauca, Colombia.

2 Corporación Colombiana de Investigación Agropecuaria (AGROSAVIA), Centro de Investigación NATAIMA, Espinal Tolima, Colombia. jmejia@agrosavia.co,julianmejia58@gmail.com (autor para correspondencia; https://orcid.org/0000-0003-0613-4504).

3 Corporación Colombiana de Investigación Agropecuaria (AGROSAVIA), Centro de Investigación Palmira. Palmira, Valle del Cauca, Colombia.galeanomendoza@gmail.com, https://orcid.org/0000-0002-5801-2421

4 Corporación Colombiana de Investigación Agropecuaria (AGROSAVIA), Centro de Investigación Motilonia. Agustín Codazzi, Cesar, Colombia.eburbano@agrosavia.co,https://orcid.org/0000-0001-5056-9893

5 Universidad Nacional de Colombia, Facultad de Ciencias Agropecuarias, Grupo de Investigación en Mejoramiento Genético, Agronomía y Producción de Semillas de Hortalizas.Palmira, Colombia. favallejoc@unal.edu.co, https://orcid.org/0000-0002-2739-0745

6 Corporación Colombiana de Investigación Agropecuaria (AGROSAVIA), Centro de Investigación la Libertad. Villavicencio, Meta, Colombia.marangoa@agrosavia.co, https://orcid.org/0000-0002-1041-072X

\section{Resumen}

Introducción. El uso de estimadores de la estabilidad para las características de interés agronómico permite conocer el comportamiento del genotipo en relación con aquellos factores del ambiente que influyen en su expresión. Objetivo. El objetivo del presente estudio fue determinar la interacción genotipo por ambiente (IGA) para seleccionar variedades de algodón con alto potencial de rendimiento de semilla y porcentaje de fibra. Materiales y métodos. Se evaluaron nueve variedades de algodón transgénico en diez localidades situadas en dos ecorregiones geográficamente diferentes: valles geográficos de los ríos Magdalena y Cauca, mediante el diseño de bloques completos al azar, entre los meses de marzo a agosto en año 2013. Resultados. El rendimiento de algodón semilla presentó IGA significativa, donde los tres primeros componentes principales (ACP) del modelo de efectos principales e interacción multiplicativa (AMMI) fueron significativos ( $<<0,01)$, con contribuciones de 86,9, 6,4 y 3,3\%, respectivamente. El rendimiento promedio para las dos ecorregiones correspondió a 3,3 t ha-1 (valle geográfico del río Magdalena) y 4,5 t ha ${ }^{-1}$ (valle geográfico del río Cauca), y para el total de las localidades dentro de las ecorregiones evaluadas correspondió a 3,9 t ha-1. El porcentaje de fibra no presentó IGA y tuvo un valor promedio de 43,34 \%. La variedad V004 presentó el mejor comportamiento con un rendimiento promedio de 4,9 t ha-1. Las variedades con mayor estabilidad fueron V001 y V002. Se observó una alta relación entre las variedades más productivas con los ambientes de mayor productividad. Conclusión. El comportamiento diferencial entre variedades y localidades para rendimiento permitió seleccionar variedades para ambientes específicos, o con plasticidad fenotípica para varios ambientes.

Palabras clave: Gossypium hirsutum, adaptación, estabilidad. 


\begin{abstract}
Introduction. The use of stability estimators for agronomic characteristics of interest allows understanding the behavior of the genotype in relation to those environmental factors that influence its expression. Objective. The objective of this study was to determine genotype by environment (GE) interaction to select cotton varieties with a high seed yield potential and fiber percentage. Materials and Methods. Nine varieties of transgenic cotton were evaluated in ten localities distributed in two geographically different ecoregions: geographical valleys of the Magdalena and Cauca rivers, by means of random complete block design, between the months of March and August in 2013. Results. Cotton seed yield had significant GE interaction, where the three principal components (PC) of the Additive Main Effects and Multiplicative Interaction (AMMI) model were significant $(\mathrm{p}<0.01)$, with contributions of $86.9,6.4$, and $3.3 \%$, respectively. The average yield for the two ecoregions were $3.3 \mathrm{t} \mathrm{ha}^{-1}$ (Magdalena river geographical valley) $4.5 \mathrm{t} \mathrm{ha}^{-1}$ (Cauca river geographical valley), and for all the evaluated localities in the ecoregions was $3.9 \mathrm{t} \mathrm{ha}^{-1}$. Fiber percentage did not present significative GE interaction and had an average value of 43,34\%. The V004 variety showed the best performance with an average yield of $4.9 \mathrm{t} \mathrm{ha}^{-1}$. The varieties with greater adaptability were V001 and V002. A high correlation was observed between the most productive varieties and the most productive environments. Conclusion. The differential behavior between varieties and localities for yield allowed to select varieties for specific environments, or with phenotypic plasticity for several environments.
\end{abstract}

Keywords: Gossypium hirsutum, adaptation, stability.

\title{
Introducción
}

El algodón se cultiva principalmente por su fibra, utilizada universalmente como materia prima de productos textiles (Mehboob-ur-Rahman et al., 2012). En el mundo se producen alrededor de veintinueve millones de toneladas de fibra, los principales productores son: China, Estados Unidos, India y Brasil (OECD y FAO, 2018). Las principales especies cultivadas en el mundo se pueden clasificar en tres grupos principales: el primer grupo lo conforman los algodones de textura media y gruesa (Gossypium hirsutum), el cual suple el $90 \%$ de la demanda mundial y es la especie más cultivada en más de cuarenta países entre las latitudes tropicales y templadas. El segundo grupo lo constituyen algodones de fibra larga, fuerte y fina, empleados para la fabricación de hilos y la manufactura de tejidos de alta calidad, pero su importancia se ha limitado a menos del $10 \%$ de la producción mundial, debido a su baja productividad (G. barbadense) (Wendel et al., 2010; Mehboob-ur-Rahman et al., 2012). Finalmente, las especies G. arboreum y G. herbaceum, las cuales se caracterizan por tener una fibra corta y gruesa, son ideales para la fabricación de tejidos pesados, muselina, relleno para muebles y algodón quirúrgico (Wendel et al., 2010).

En Colombia la producción de algodón ha decrecido significativamente de 28000 ha en 1970 a tan solo 10 000 ha en el año 2016, esto se debe principalmente a problemas sanitarios, así como a la importación de algodón de bajo precio e inferior calidad (CONALGODÓN, 2019). Adicionalmente, la producción nacional de algodón es afectada por los altos costos de producción y los bajos rendimientos, lo que genera importaciones desde países como Argentina y Estados Unidos (Martínez-Reina y Hernández, 2015). Por tanto, es necesario evaluar materiales mejorados con altos rendimientos y buena calidad de fibra, estables y/o con adaptaciones específicas a cada una de las zonas productoras.

Es importante determinar el comportamiento diferencial de los genotipos, o el grado de interacción genotipo ambiente (IGA); comportamiento que se manifiesta independientemente, según el grado de presencia de interacción en cada uno de los ambientes particulares donde son evaluados estos genotipos, a su vez, es necesario poder determinar cuáles genotipos presentan el menor grado de esta interacción, sin ir en detrimento del rendimiento, 
lo que se considera como estables (González et al., 2007). Para la definición de los estimadores de la estabilidad se han utilizados diferentes metodologías tanto univariadas como multivariadas. Los estudios unvariados con diferentes métodos aplicados y comparados por González et al. (2010), a su vez en estudios realizados por Vargas et al. (2016), en donde se evaluaron maíces híbridos amarillos QPM en diferentes localidades, y a su, se realizó un análisis multivariado de efectos principales aditivos e interacciones multiplicativas (AMMI); con estos métodos los autores lograron determinar la estabilidad de los maíces evaluados en las principales zonas productoras del cultivo en Colombia. Los métodos un variados emplean la media aritmética, también el coeficiente de regresión, a su vez correlacionan los genotipos con los índices ambientales, midiendo el grado o magnitud de la estabilidad de acuerdo con la magnitud de la desviación con respeto a la regresión (Rueda y Cotes, 2009). En el modelo AMMI, es uno de los métodos multivariados empleados, en donde los efectos de los genotipos y los ambientes se asumen como lineales, explicados por el análisis de varianza y, a su vez la interacción o parte multiplicativa entre ellos, bien explicada por el análisis de componentes principales en un Biplot (Rodríguez-Pérez et al., 2005). La especificidad o plasticidad de un nuevo cultivar de algodón depende del genotipo, del ambiente y de la interacción enre ambos (Ali et al., 2005). Adicionalmente, el rendimiento de una variedad depende en mayor proporción de la genética con contribuciones cercanas al $50 \%$, tanto para el manejo como para la interacción de esta con el ambiente (Bednarz et al., 2000; Liu et al., 2013; Zhi et al., 2016). Por tanto, la habilidad de los genotipos para responder de manera diferencial en ambientes contrastantes implica modificaciones fisiológicas, metabólicas y de crecimiento, que pueden estar directamente relacionadas a la respuesta a estreses bióticos o abióticos (Des-Marais et al., 2013).

En la mejora genética es de interés la selección de genotipos de elevada productividad y de buen comportamiento en diversos ambientes (Bednarz et al., 2000; Liu et al., 2013; Orawu et al., 2017). La presencia de la interacción provoca un aumento en el desvío del parentesco fenotípico, reducción de la heredabilidad a lo largo de los ambientes y, consecuentemente disminución de las ganancias genéticas potenciales (Mudada et al., 2017). Por tanto, descartar material con alta interacción en pro de minimizar los efectos ambientales es necesario dentro de los programas de mejoramiento, los cuales incluyen evaluaciones en múltiples ambientes para asegurar el mejor comportamiento de los posibles nuevos cultivares (Bernardo, 2016). El objetivo del presente estudio fue determinar la interacción genotipo por ambiente (IGA) para seleccionar variedades de algodón con alto potencial de rendimiento de algodón semilla y porcentaje de fibra.

\section{Materiales y métodos}

La evaluación de las variedades de algodón (Gossypium hirsutum) (Cuadro 1) se realizó en diez localidades distribuidas en dos ecorregiones (cinco en cada una) de Colombia: valle geográfico del río Cauca y valle geográfico del río Magdalena (Cuadro 2). La evaluación se realizó entre los meses de marzo y agosto del 2013.

Los materiales evaluados correspondieron a nueve variedades transgénicas de algodón provenientes de diferentes empresas privadas, todas mejoradas y procedentes de Estados Unidos (Cuadro 1). En el ensayo se utilizaron testigos comerciales liberados y comercializados, para el momento del estudio, en las zonas agroecológicas de Colombia. Para el establecimiento de los tratamientos, en cada una de las localidades se utilizó el diseño de bloques completos al azar (BCA), con cuatro repeticiones y nueve tratamientos. Las unidades experimentales estuvieron conformadas por ocho surcos de $10 \mathrm{~m}$ lineales de longitud, y una distancia de $0,90 \mathrm{~m}$ entre surcos. Se sembraron inicialmente 150 semillas en los $10 \mathrm{~m}$, con una densidad efectiva de seis a siete plantas después del raleo. No se hicieron aplicaciones de herbicidas antes de la siembra, y los materiales se condujeron de acuerdo con el manejo agronómico de algodón convencional para cada una de las zonas en estudio. Se midieron las variables: rendimiento de algodón semilla (R), el cual correspondió a la cosecha de los cuatro surcos centrales efectivos, expresado en kg ha-1 , y el porcentaje de fibra (\% FB), para la cual se cosecharon cincuenta motas por unidad experimental del tercio medio de las plantas 
Cuadro 1. Variedades de algodón transgénico* (Gossypium hirsutum) evaluadas en diez localidades, de dos ecorregiones: valles geográficos de los ríos Magdalena y Cauca, Colombia. 2013.

Table 1. Transgenic* cotton (Gossypium hirsutum) varieties evaluated in ten localities from two ecoregions: geographic valleys of Magdalena and Cauca rivers, Colombia. 2013.

\begin{tabular}{cll}
\hline Variedades & Duración ciclo & Características de las variedades \\
\hline V001+ & Intermedio & Tolerancia herbicidas glifosato y Glufosinato \\
V002+ & Intermedio & Tolerancia a glifosato y control de principales lepidópteros \\
V003+ & Intermedio & Tolerancia a glifosato y control de principales lepidópteros \\
V004+ & Intermedio & Tolerancia a glifosato y control de principales lepidópteros \\
V005+ & Intermedio & Tolerancia a glifosato y control de principales lepidópteros (testigo) \\
V006+ & Intermedio & Tolerante herbicida Glufosinato (testigo) \\
V007* & Intermedio & Tolerante herbicida Glifosato (testigo) \\
V008* & Intermedio & Tolerancia a glifosato y control de principales lepidópteros (testigo) \\
V009* & Intermedio & Tolerancia a glifosato y control de principales lepidópteros \\
\hline
\end{tabular}

Compañías dueñas de las variedades, *Monsanto y +Bayer (Ahora BASF) / Varieties owned by *Monsanto and +Bayer (currently BASF).

Cuadro 2. Características climáticas y edafológicas de las diez localidades de dos ecorregiones: valles geográficos de los ríos Magdalena y Cauca, Colombia. 2013.

Table 2. Climatic and edaphological characteristics of the ten locations from two ecoregions: geographic valleys of Magdalena and Cauca rivers, Colombia. 2013.

\begin{tabular}{|c|c|c|c|c|c|c|c|c|}
\hline \multirow[t]{2}{*}{ Localidad } & \multirow[t]{2}{*}{ Longitud } & \multirow[t]{2}{*}{ Altitud } & \multirow{2}{*}{$\begin{array}{c}\text { T media } \\
\left({ }^{\circ} \mathbf{C}\right)\end{array}$} & \multirow{2}{*}{$\begin{array}{l}\text { HR } \\
(\%)\end{array}$} & \multirow[t]{2}{*}{$\mathbf{B S}^{\mathbf{a}}$} & \multirow[t]{2}{*}{ Precipitación } & \multicolumn{2}{|c|}{ Suelo } \\
\hline & & & & & & & Textura $^{\mathrm{b}}$ & Fertilidad \\
\hline A. Armero & $04^{\circ} 53^{\prime} 52.2^{\prime \prime} \mathrm{N}$ & $074^{\circ} 50^{\prime} 28.4^{\prime \prime} \mathrm{W}$ & 28,6 & 72,3 & 1338,8 & 856,1 & $\mathrm{Ff} / \mathrm{A}$ & Alta \\
\hline B. Ambalema & $04^{\circ} 49^{\prime} 05.8^{\prime \prime} \mathrm{N}$ & $074^{\circ} 45^{\prime} 14.5^{\prime \prime} \mathrm{W}$ & 26,2 & 70 & 618,1 & 723,3 & $\mathrm{Ff} / \mathrm{A}$ & Media \\
\hline C. Espinal & $04^{\circ} 22^{\prime} 1^{\circ} .34^{\prime \prime} \mathrm{N}$ & $074^{\circ} 91^{\prime} 23.2^{\prime \prime} \mathrm{W}$ & 29,2 & 75 & 945,3 & 1083,2 & $\mathrm{Ff} / \mathrm{A}$ & Media \\
\hline D. Guamo & $04^{\circ} 11^{\prime} 29.1^{\prime \prime} \mathrm{N}$ & $075^{\circ} 00^{\prime} 46.6^{\prime \prime} \mathrm{W}$ & 28,6 & 75,1 & 953,8 & 1005,6 & $\mathrm{Ff} / \mathrm{A}-\mathrm{Ar} / \mathrm{A}$ & Media \\
\hline E. Palermo & $02^{\circ} 50^{\prime} 31.9 ” \mathrm{~N}$ & $075^{\circ} 18^{\prime} 52.1^{\prime \prime} \mathrm{W}$ & 29,7 & 65 & 1951,4 & 1580,7 & $\mathrm{Ar} / \mathrm{A}$ & Media \\
\hline F. Andalucía & $04^{\circ} 10^{\prime} 03.1{ }^{\prime \prime} \mathrm{N}$ & $076^{\circ} 11^{\prime} 07.02^{\prime \prime} \mathrm{W}$ & 23,5 & 72,3 & 1338,8 & 1365,5 & T.Fina & Media \\
\hline G. Candelaria & $03^{\circ} 24^{\prime} 24.0^{\prime \prime} \mathrm{N}$ & $076^{\circ} 18^{\prime} 00.9^{\prime \prime} \mathrm{W}$ & 27,3 & 69 & 618 & 1080,0 & Ff/A-Ff/frag. & Alta \\
\hline H. El Cerrito & $03^{\circ} 41^{\circ} 28.4^{\prime \prime} \mathrm{N}$ & $076^{\circ} 18^{\prime} 31.3^{\prime \prime} \mathrm{W}$ & 24,1 & 75 & 945,3 & 1021,8 & T.fina & Media \\
\hline I. Palmira & $03^{\circ} 31^{\prime} 01.0^{\prime \prime} \mathrm{N}$ & $076^{\circ} 25^{\prime} 35.3^{\prime \prime} \mathrm{W}$ & 24,7 & 75,1 & 953,8 & 1008,6 & Ff/A-Ff/frag & Media \\
\hline J. Viges & $02^{\circ} 50^{\prime} 31.9 ” \mathrm{~N}$ & $075^{\circ} 18^{\prime} 52.1^{\prime \prime} \mathrm{W}$ & 24,7 & 65 & 1951,4 & 1288,1 & A-A/arc. & Alta \\
\hline
\end{tabular}

a BS: brillo solar horas, es el tiempo en el cual incide luz solar directa sobre alguna localidad, entre el alba y el atardecer / a BS: sunshine duration, is the time in which direct sunlight falls on some location, between sunrise to sunset.

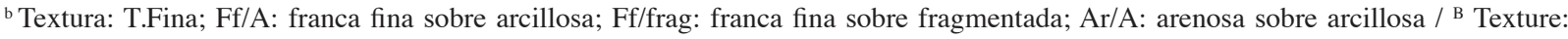
T.fine; Ff/A: clay loam; Ff/frag: coarse loam: Ar/A: sandy clay.

dentro de los cuatro surcos centrales, antes de la cosecha total de la unidad experimental, las cuales se desmotaron para determinar el \% de fibra total recolectado por genotipo de acuerdo con la relación.

El análisis estadístico se realizó usando el modelo AMMI (Additive Main Effects and Multiplicative Interaction) (Oliveira et al., 2014) y la librería Agricolae (de-Mendiburu, 2019) del software estadístico R (R Core 
Team, 2015). La estimación de la interacción genotipo por ambiente (IGA) se realizó como la describió Oliveira et al. (2014) [1]. Adicionalmente, se realizó la prueba de Gollob con el fin de reducir la dimensionalidad de los componentes principales (CPs) (Lozano-Ramírez et al., 2015):

$$
Y_{i j k}=\mu+G_{i}+E_{j}+B(E)_{k(j)}+\sum_{n=1}^{N} \lambda_{n} \gamma_{i n} \delta_{j n}+\rho_{(i j)}+e_{i j}
$$

$Y_{i j k}=$ observación $k$ del genotipo $i$ en el ambiente $j$.

$\mu=$ media general.

$G_{i}=$ efecto principal del genotipo $i$ (considerado fijo).

$E_{j}=e$ fecto principal del ambiente $j$ (considerado aleatorio).

$B(E)_{k(j)}=$ efecto del bloque $K$ dentro del ambiente $j$.

$\lambda_{k}=$ es el valor singular del eje $\mathrm{K}$ en el análisis de componentes principales.

$\gamma_{i k}, \delta_{j k}=$ son los factores genotípicos y ambientales, respectivamente, de los vectores singulares asociados con $\lambda_{k}$ $N$ = Número de componentes principales retenidos en el modelo.

$\rho_{(\mathrm{ij})}=$ residual de la de la interaccion GE.

$e_{(i j)}=$ errorasociado con cada medición.

\section{Resultados}

Las fuentes de variación correspondiente a localidades, genotipos y la IGA, fueron altamente significativas $(\mathrm{p} \leq 0,01)$ para la variable rendimiento de algodón semilla. Existió un comportamiento diferencial de las variedades en cada uno de los ambientes en los cuales fueron evaluadas (Cuadro 3). En contraste, para la variable porcentaje de fibra no se observaron diferencias significativas entre variedades, ni para la interacción de estas con el ambiente; sin embargo, se presentaron diferencias significativas entre localidades.

Cuadro 3. Cuadrados medios para las variables rendimiento y porcentaje de fibra $(\% \mathrm{FB})$, de nueve variedades transgénicas de algodón (Gossypium hirsutum) evaluadas en dos ecorregiones: valles geográficos de los ríos Magdalena y Cauca, Colombia. 2013.

Table 3. Mean square for cotton-seed yield and fiber percentage (\%FB) of nine genetically modified cotton (Gossypium hirsutum) varieties evaluated in two ecoregions: geographic valleys of Magdalena and Cauca rivers, Colombia. 2013.

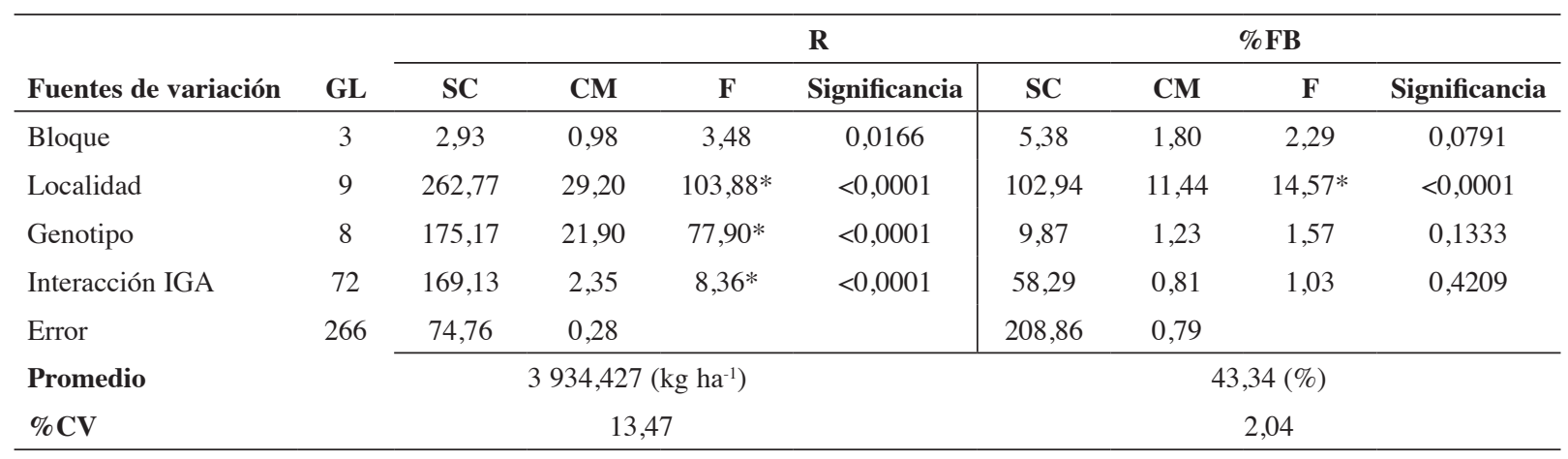

* Significancia estadística con $\mathrm{p} \leq 0,01 / *$ Statistically significance with $\mathrm{p} \leq 0.01$. 
La contribución relativa de cada fuente de variación al total de la variabilidad encontrada para rendimiento (R) y porcentaje de fibra (\%FB) estuvo fraccionada (Cuadro 4), lo que muestra una mayor contribución total ejercida por el ambiente (A), con niveles superiores al $50 \%$ de la variación total explicada. Las demás fuentes de variación en la variable rendimiento $(\mathrm{R})$, también presentaron significancia y aportaron una menor contribución, del 41 $\%$ para genotipo $(\mathrm{G})$ y $4,4 \%$ para la IGA. El carácter porcentaje de fibra $(\% \mathrm{FB})$ no mostró significancia en sus distintas fuentes de variación (Cuadro 4), con excepción del ambiente (localidad).

Cuadro 4. Análisis de varianza y proporción de la variación explicada por genotipo (G), ambiente (A) y la interacción entre ambos (IGA) para rendimiento de algodón (Gossypium hirsutum) y porcentage de fibra en nueve variedades transgénicas, evaluadas en dos ecorregiones: valles geográficos de los ríos Magdalena y Cauca, Colombia. 2013.

Table 4. Analysis of variance and variance proportion explained by genotype (G), environment (E) and interaction between both (IGA) for cotton (Gossypium hirsutum) seed yield and percentage of fiber of nine genetically modified varieties evaluated in two ecoregions: geographic valley of Magdalena and Cauca rivers. Colombia. 2013.

\begin{tabular}{|c|c|c|c|c|c|c|}
\hline \multirow[b]{2}{*}{ Caracteres } & \multicolumn{2}{|c|}{ Genotipo $(\mathbf{G})$} & \multicolumn{2}{|c|}{ Ambiente (A) } & \multicolumn{2}{|c|}{ IGA } \\
\hline & CM & $\begin{array}{c}\text { Proporción } \\
\text { varianza }\end{array}$ & $\mathbf{C M}$ & $\begin{array}{c}\text { Proporción } \\
\text { varianza }\end{array}$ & $\mathbf{C M}$ & $\begin{array}{c}\text { Proporción } \\
\text { varianza }\end{array}$ \\
\hline Rendimiento & $21,90 *$ & $41,0 \%$ & $29,20 *$ & $54,6 \%$ & $2,35^{*}$ & $4,4 \%$ \\
\hline Fibra (\%) & $1,23 \mathrm{~ns}$ & $9,1 \%$ & $11,44^{*}$ & $84,90 \%$ & $0,81 \mathrm{~ns}$ & $6,0 \%$ \\
\hline
\end{tabular}

* Diferencia estadística significativa a nivel del 0,1\%. ns: diferencia estadística no significativa. CM: cuadrado medio para cada uno de los componentes de variación / * Statistically significant difference at $0.1 \%$. ns: non-significant difference; CM: mean square of each variation components.

El modelo presentó un coeficiente de variación de $13,47 \%$ para rendimiento y $2 \%$ para porcentaje de fibra (\%FB), lo que indica confiabilidad y reproducibilidad de la evaluación. El rendimiento (R) promedio del experimento fue de $3,9 \mathrm{tha}^{-1}$, con valores medios por localidad que van desde 2,7 hasta 4,8 $\mathrm{t} \mathrm{ha}^{-1}$ para las localidades de Espinal y Candelaria, respectivamente.

Producto de la descomposición del valor singular para reducir la dimensionalidad en los componentes principales, se realizó la prueba de Gollob con el objeto de determinar la significancia de cada componente (Lozano-Ramírez et al., 2015). Se observó que los tres primeros componentes principales C1, C2 y C3 presentaron significancia estadística $(\mathrm{p}>0,01)$ para el carácter rendimiento en $\mathrm{t}$ ha ${ }^{-1}(\mathrm{R})$ con una contribución de 86,9,6,4 y 3,3, y con 16,14 y 12 grados de libertad, respectivamente. Los dos primeros componentes principales (CP) explicaron el 93,3\% de la variabilidad total observada (Cuadro 5).

El análisis AMMI permitió realizar agrupaciones de ambientes (macroambientes). El primero correspondió a la ecorregión del valle del Cauca con sus correspondientes localidades (Figura 1). Específicamente, la localidad Vijes fue la más contrastante en relación con las demás localidades de la misma ecoregión, al presentar el mayor potencial productivo, con 5,1 t ha-1 y diferente a los demás ambientes presentes (Cuadro 6), lo anterior debido a que en esta ecorregión se presentaron mayores precipitaciones para el general de las localidades evaluadas, a su vez menores temperaturas (Cuadro 2), los suelos de esta ecorregión presentaron mejores características; sin embargo, no hubo una asociación específica con algún genotipo, por tanto, esta localidad es un ambiente en donde se podría realizar evaluaciones de productividad en los procesos de selección de nuevas variedades de algodón, donde expresaran su máximo potencial productivo. Dentro de esa ecorregión las localidades de Palmira, El Cerrito y Candelaria conforman el macro ambiente valle del Cauca, con producciones promedios de 4,9, 4,6 y 4,6 t ha ${ }^{-1}$, respectivamente. Este valle, presentó la mejor opción agroclimática para el rendimiento de los materiales evaluados (Cuadro 6). 
Cuadro 5. Prueba de Gollob para los factores en el modelo AMMI, en la variable rendimiento de nueve variedades transgénicas de algodón (Gossypium hirsutum), evaluadas en dos ecorregiones: valles geográficos de los ríos Magdalena y Cauca, Colombia. 2013. Table 5. Gollop test for the factors in the AMMI model, in the variable yield of nine transgenic cotton (Gossypium hirsutum) varieties evaluated in two ecoregions: geographic valleys of Magdalena and Cauca rivers, Colombia. 2013.

\begin{tabular}{ccccccc}
\hline Componente & SS & PORCENT & PORCENAC & DF & MS & F \\
\hline C1 & 99,595 & 86,9171 & 86,917 & 16 & 18,7247 & $66,616^{*}$ \\
C2 & 22,378 & 6,4923 & 93,409 & 14 & 1,5985 & $5,6868^{*}$ \\
C3 & 11,412 & 3,3108 & 96,72 & 12 & 0,9510 & $3,3833^{*}$ \\
C4 & 6,605 & 1,9161 & 98,636 & 10 & 0,6605 & 2,3498 \\
C5 & 3,121 & 0,9056 & 99,542 & 8 & 0,3902 & 1,3881 \\
C6 & 0,929 & 0,2694 & 99,811 & 6 & 0,1548 & 0,5506 \\
C7 & 0,541 & 0,157 & 99,968 & 4 & 0,1353 & 0,4814 \\
C8 & 0,105 & 0,0306 & 99,999 & 2 & 0,0527 & 0,1874 \\
C9 & 0,004 & 0,0011 & 100 & 0 & & . \\
\hline
\end{tabular}

SS: suma de cuadrados, PORCENT: proporción de la variabilidad explicada para cada término, PORCENAC: proporción de variabilidad acumulada explicada por cada término, DF: grados de libertad para cada componente, MS: cuadrado medio de los componentes principales, F: valor de prueba f. * Significancia estadística con $\mathrm{p} \leq 0,01$ / SS: sum of squares, PORCENT: proportion of the variability explained by each component, PORCENAC: proportion of cumulative variability explained by each component, DF: degrees of freedom for each component, MS: mean square of the main components, F: F-test value. * Statistical significance difference $\mathrm{p} \leq 0.01$.

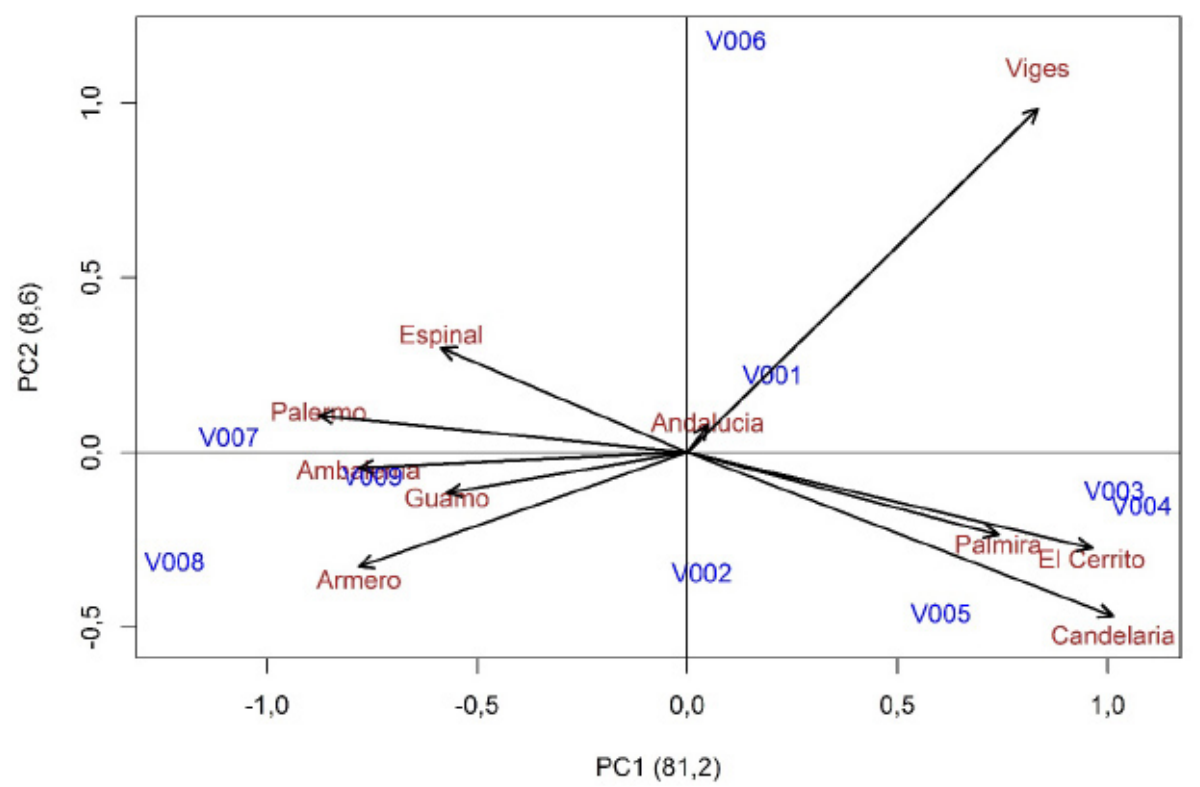

Figura 1. Biplot para la descomposición de la interacción genotipo ambiente para la variable rendimiento de semilla, en nueve variedades de algodón transgénico (Gossypium hirsutum), evaluadas en diez localidades de dos ecorregiones: valles geográficos de los ríos Magdalena y Cauca, Colombia. 2013.

Figure 1. Biplot for the decomposition of the genotype-environment interaction for the variable seed yield in nine transgenic cotton (Gossypium hirsutum) varieties evaluated in ten localities of two ecoregions: geographic valleys of Magdalena and Cauca rivers, Colombia. 2013. 
Cuadro 6. Medias estimadas para la variable rendimiento de semillas ( $\left.\mathrm{t} \mathrm{ha}^{-1}\right)$ del modelo AMMI en nueve variedades de algodón transgénico (Gossypium hirsutum), evaluadas en dos ecorregiones: valles geográficos de los ríos Magdalena y Cauca, Colombia. 2013.

Table 6. Estimated mean cotton seed yield values ( $\mathrm{t} \mathrm{ha} \mathrm{h}^{-1}$ ) from the AMMI model of nine genetically modified cotton (Gossypium hirsutum) varieties evaluated in two ecoregions: geographic valleys of Magdalena and Cauca rivers, Colombia. 2013.

\begin{tabular}{|c|c|c|c|c|c|c|c|c|c|c|}
\hline \multirow[t]{2}{*}{ Ambiente } & \multicolumn{10}{|c|}{ Genotipo } \\
\hline & V008 & V009 & V007 & V004 & V003 & V005 & V001 & V006 & V002 & Promedio \\
\hline Andalucía & 2,6 & 2,4 & 2,1 & 4,2 & 3,8 & 4,2 & 3,0 & 3,2 & 3,3 & 3,2 bc \\
\hline Candelaria & 3,2 & 3,0 & 2,7 & 6,8 & 6,5 & 6,6 & 4,9 & 4,2 & 5,3 & $4,8 \mathrm{a}$ \\
\hline El Cerrito & 3,2 & 3,1 & 2,6 & 6,8 & 6,3 & 6,5 & 4,8 & 4,5 & 5,3 & $4,8 \mathrm{a}$ \\
\hline Palmira & 3,3 & 3,4 & 2,6 & 6,4 & 6,3 & 5,6 & 4,7 & 4,3 & 5,5 & $4,7 \mathrm{a}$ \\
\hline Vijes & 3,1 & 3,3 & 3,1 & 6,8 & 6,4 & 5,5 & 5,4 & 6,3 & 5,5 & 5,1 a \\
\hline Promedio & & & & & & & & & & 4,5 \\
\hline Ambalema & 3,6 & 3,1 & 3,1 & 3,4 & 3,1 & 3,7 & 2,9 & 3,1 & 3,9 & $3,3 \mathrm{~b}$ \\
\hline Armero & 3,7 & 3,3 & 2,8 & 3,6 & 3,2 & 3,3 & 3,0 & 2,6 & 4,2 & $3,3 \mathrm{bc}$ \\
\hline Espinal & 2,9 & 2,2 & 2,1 & 2,7 & 2,7 & 3,4 & 2,7 & 2,9 & 2,3 & $2,7 \mathrm{c}$ \\
\hline Guamo & 5,0 & 2,9 & 4,4 & 5,1 & 4,5 & 4,5 & 4,1 & 4,2 & 5,3 & $4,5 \mathrm{a}$ \\
\hline Palermo & 3,4 & 2,9 & 2,9 & 2,8 & 2,8 & 3,3 & 2,9 & 3,0 & 3,5 & 3,0 bc \\
\hline Promedio & & & & & & & & & & 3,3 \\
\hline Promedio & 3,4 bc & $3,0 \mathrm{c}$ & 2,9 c & $4,9 \mathrm{a}$ & $4,6 \mathrm{a}$ & $4,6 \mathrm{a}$ & $3,9 \mathrm{ab}$ & 3,8 abc & $4,4 \mathrm{ab}$ & 3,9 \\
\hline
\end{tabular}

La segunda ecorregión correspondiente al valle geográfico del río Magdalena presentó, en general, una baja productividad. Esta ecorregión, correspondiente a los departamentos del Tolima y Huila, los cuales se caracterizan por presentar promedios más altos de temperatura con diferencia de $5{ }^{\circ} \mathrm{C}$ por encima del departamento del valle del Cauca y a su vez, menor humedad relativa (HR), suelos con texturas francas (livianas), menor brillo solar (BS) y precipitación, lo cual limita la expresión del potencial productivo del algodón; y da como resultado menores rendimientos (Cuadro 6). La localidad con un potencial productivo de 4,5 t ha-1 en esta zona correspondió al municipio del Guamo, los demás ambientes presentaron menores producciones, inferiores a $3 \mathrm{t} \mathrm{ha}^{-1}$ (Cuadro 6). Las localidades de Palermo, Ambalema, Guamo y Armero están directamente asociadas a las variedades V007, V009 y V008, que corresponden a materiales del programa de mejoramiento de la multinacional Monsanto (Cuadro 2), el resto de las variedades corresponden al programa de mejoramiento de Bayer (Ahora BASF). Se asocian las variedades de mayor productividad, V003, V004 y V005, a Palmira, El Cerito y Candelaria, ambientes de mayor productividad, a su vez las variedades V007, V009 y V008 a los ambientes de menor potencial productivo.

La localidad de Andalucía no presentó influencia sobre la expresión de los genotipos evaluados, y careció de correlación con las demás localidades, con un potencial productivo de 3,2 $\mathrm{t} \mathrm{ha}^{-1}$ (Figura 1). A su vez la variedad V004 presentó el mejor comportamiento con un rendimiento promedio de 4,9 $\mathrm{tha}^{-1}$. De forma similar, la variedad V006 careció de asociación con los ambientes evaluados en el presente estudio.

\section{Discusión}

La IGA significativa en este estudio, como resultado de materiales y ambientes contrastantes, permite inferir que el rendimiento fue una característica compleja, poligénica y de baja heredabilidad; sin embargo, cuando los ambientes no son muy contrastantes o los materiales han sido previamente seleccionados por su plasticidad, no se 
encuentran diferencias significativas para la IGA, pero sí se puede encontrar significancia en el comportamiento de cada población en un ambiente específico (Mudada et al., 2017). Resultados similares han sido reportados para algodón, en donde el rendimiento y calidad de la fibra están directamente asociados con ambientes específicos (Greveniotis y Sioki, 2017).

Los valores reportados en esta investigación están dentro del rango esperado para materiales élite mejorados y evaluados en ambientes contrastantes (Mudada et al., 2017).

La mayor contribución del ambiente a la variabilidad total indica que las mejores condiciones climáticas y edafológicas incidieron directamente en la expresión de los caracteres (Ali et al., 2005). De forma similar se han encontrado diferencias significativas en el porcentaje de fibra asociado al riego y las dosis de fertilización nitrogenada, pero no para interacción de las variables (Palomo et al., 2004). Al evaluar materiales de diferente origen y grupo de madurez en localidades contrastantes, se presentó una IGA significativa para los parámetros de porcentaje y calidad de fibra (Greveniotis y Sioki, 2017).

Las variedades V002 y V001 con valores cercanos al vértice 0 en el biplot, presentaron alta estabilidad entre las localidades evaluadas con baja interacción con el ambiente, y adecuado comportamiento en rendimiento de algodón semilla, con valores promedio de 4,4 y 3,9 ha- ${ }^{-1}$, respectivamente. Estas variedades no presentaron un asocio con un ambiente específico, sin embargo, pueden crecer en los ambientes de evaluación y mostrar buenos resultados (Cuadro 6 y Figura 1).

Los materiales más estables generalmente no son los más productivos, puesto que existe un ligamiento de genes entre alta productividad y especificidad o presencia de interacción. Por ejemplo, en una evaluación multiambiental en maíz los promedios de los mejores híbridos estuvieron en el rango de 6,9 a 7,1 t ha-1; sin embargo, los genotipos más estables producen alrededor de 6,3 $\mathrm{t} \mathrm{ha}^{-1}$ (Lozano-Ramírez et al., 2015). La estabilidad fenotípica de un material es una característica genética aditiva, por tanto, los materiales que presentan alta estabilidad a través de varios ambientes muy probablemente tiene una baja IGA (Vargas et al., 2016). Adicionalmente, muchos de los mecanismos genéticos y epigenéticos que regulan la estabilidad de un genotipo, también están relacionados con la respuesta a factores de estreses bióticos y abióticos, por tanto, al seleccionar materiales con baja IGA indirectamente se pueden estar seleccionado materiales con tolerancia a calor o a sequía (Des-Marais et al., 2013; Sareen et al., 2014). Sin embargo, los materiales con alta IGA con especificidad a determinadas localidades, generalmente tienen mejores rendimientos y responden mejor a las condiciones agronómicas o ambientes favorables, pero con baja estabilidad ambiental.

La selección de localidades es importante en los programas de mejoramiento, por ello, se debe realizar en las zonas más representativas dentro de un macroambiente las primeras selecciones de progenies, y posteriormente, las localidades más discriminantes se utilizan con las líneas avanzadas para evaluar su estabilidad (Orawu et al., 2017).

En general, las variedades de algodón transgénico respondieron a mejores condiciones de suelos presentes en el departamento del valle del Cauca (Cuadro 2), donde se tuvo una respuesta superior en términos de productividad (4,5 y 3,3 t ha $^{-1}$ para valle del Cauca y valle del Magdalena, respectivamente). Por tanto, el modelo AMMI permitió analizar el rendimiento de algodón semilla e identificar los mejores genotipos y los mejores ambientes (LozanoRamírez et al., 2015). Los genotipos V002 y V001 se podrían recomendar para validarlos en las dos subregiones agroecológicas por su aparente estabilidad. Se asociaron las variedades de mayor productividad (V003, V004 y V005) a Palmira, El Cerito y Candelaria, ambientes de mayor productividad, a su vez las variedades V007, V009 y V008 a los ambientes de menor potencial productivo (Figura 2).

El modelo AMMI permitió agrupar los ambientes en dos grupos bien marcados y contrastantes, el primero con materiales de alta productividad, asociados a ambientes con buenas características edafológicas (valle del Cauca) y el segundo ambiente con genotipos de menor potencial correlacionados con menores valores edafológicos (valle del río Magdalena) (Cuadro 1). La ecorregión del valle geográfico del río Magdalena también tuvo un efecto marcado en híbridos mejorados de maíz, donde la localidad de Espinal fue donde se presentó el menor rendimiento (Vargas 


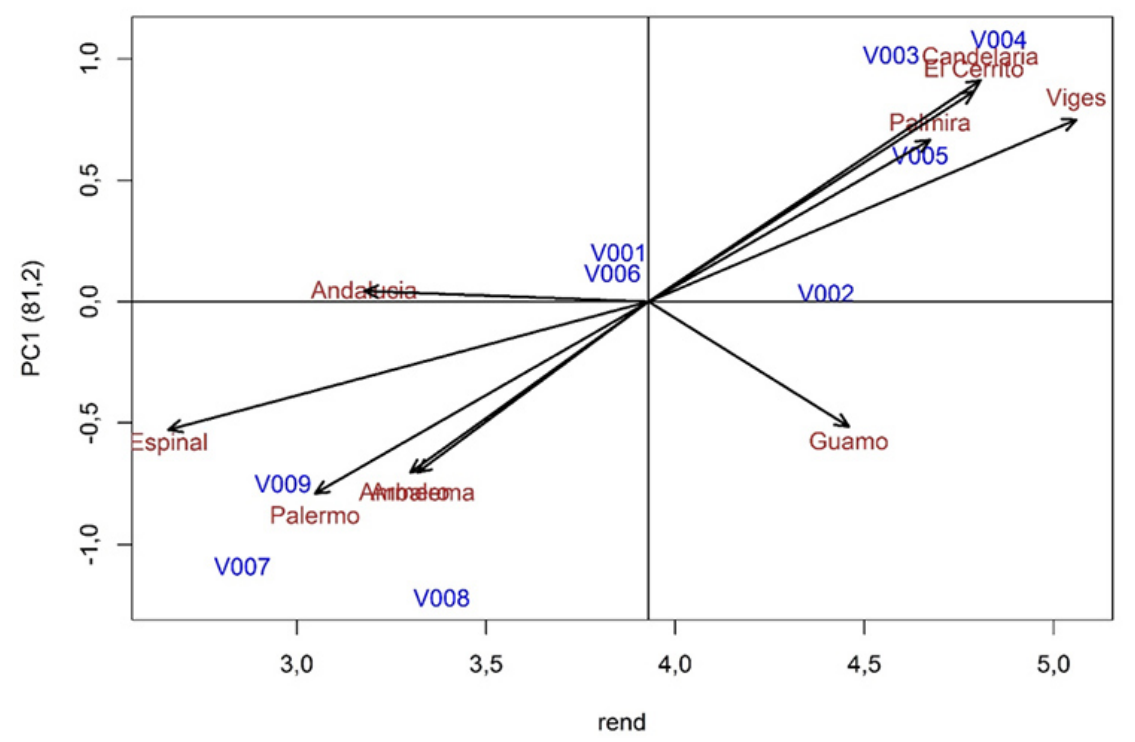

Figura 2. Biplot (Rendimiento de semilla de algodón -rend- vs Componente principal 1) para la descomposición de la interacción genotipo ambiente para la variable rendimiento de semilla, en nueve variedades de algodón transgénico, evaluadas en diez localidades de dos ecorregiones: valles geográficos de los ríos Magdalena y Cauca, Colombia. 2013.

Figure 2. Biplot (cotton seed yield -rend- vs maincomponent 1) for the decomposition of the genotype environment interaction in function of cotton seed yield from nine transgenic cotton varieties evaluated in ten localities of two ecoregions: geographic valleys of Magdalena and Cauca rivers, Colombia. 2013.

et al., 2016). Los resultados del presente estudio son importantes para la planeación de los procesos de selección de genotipos para adaptaciones amplias y específicas a las diferentes zonas productivas del país (Williams et al., 2010). Además, la identificación de macro ambientes y ambientes contrastantes facilita la evaluación y selección de progenies disminuyendo el efecto ambiental (Crossa, 2012) o determinar aquellos genotipos que se adapten a diferentes ambientes y efectos potenciales del cambio climático.

\section{Conclusiones}

Mediante la evaluación de la IGA sobre caracteres cuantitativos, se observaron las respuestas diferenciales de estas variedades transgénicas a los diferentes ambientes, determinando con precisión el grado de adaptación a los ambientes específicos evaluados. Se encontró una fuerte asociación entre las variedades más productivas con los ambientes de mayor producción y oferta edafoclimática, a su vez, se determinó el agrupamiento en sus macro ambientes contrastantes. Se sugiere validar las variedades V001 y V002 en los dos ambientes evaluados, por su estabilidad presentada y aceptable comportamiento, mientras que las variedades más productivas (V003 V004 y V005) se recomienda validarlas para el macroambiente del valle del Cauca. Variedades asociadas al valle geográfico del río Magdalena (V007, V008 y V009), presentaron los menores rendimientos; sin embargo, no se observaron diferencias estadísticas entre genotipos, a su vez, tampoco para la IGA en el carácter porcentaje de fibra (\% FB), lo que mostró que todos los genotipos presentaron comportamientos similares en este aspecto. 


\section{Agradecimientos}

Los autores expresan su agradecimiento a Semillas del Valle S.A. por el apoyo financiero y logístico para realizar esta investigación, y a la Corporación Colombiana de Investigación Agropecuaria (AGROSAVIA).

\section{Literatura citada}

Ali, Y., Z. Aslam, and F. Hussain. 2005. Genotype and environment interaction effect on yield of cotton under naturally salt stress condition. Int. J. Environ. Sci. Technol. 2:169-173. doi:10.1007/BF03325872

Bednarz, C.W., D.C. Bridges, and S.M. Brown. 2000 Analysis of cotton yield stability across population densities. Agron. J. 92:128-135. doi:10.2134/agronj2000.921128x

Bernardo, R. 2016. Bandwagons I, too, have known. Theor. Appl. Genet. 129:2323-2332. doi:10.1007/s00122-016-2772-5

Castro, F., D. Contreras, L. Tamayo, y L. Trujillo. 2013. Análisis de la competitividad de la cadena algodón, fibras, textiles y confecciones. FEDESARROLLO, Bogota, COL. https://www.repository.fedesarrollo.org.co/bitstream/handle/11445/204/ Analisis\%20cadena\%20algodon.pdf?sequence=2\&isAllowed=y (consultado set. 2018).

CONALGODÓN (Confederación Colombiana del Algodón). 2019. Estadísticas. CONALGODON, COL. http://conalgodon. com/estadisticas/\#sc-tabs-1475792539449 (consultado set. 2018).

Crossa, J. 2012. From genotype $\times$ environment interaction to gene $\times$ environment interaction. Curr. Genomics 13:225-244. doi: $10.2174 / 138920212800543066$

De-Mendiburu, F. 2019. Package 'agricolae'. R Project. https://cran.r-project.org/web/packages/agricolae/agricolae.pdf (accessed Apr. 2019).

Des-Marais, D.L., K.M. Hernandez, and T.E. Juenger. 2013. Genotype-by-environment interaction and plasticity: exploring genomic responses of plants to the abiotic environment. Annu. Rev. Ecol. Evol. Syst. 44:5-29. doi:10.1146/annurevecolsys-110512-135806

González, A., D. Pérez, J. Sahagún, O. Franco, E.J. Morales, M. Rubi, F. Gutiérrez, y A. Balbuena. 2010. Aplicación y comparación de metodos univariados para evaluar la estabilidad en amices del Valle Toluca Atlacomulco, México. Agron. Costarricense 34(2):129-143.

González, T., E. Monteverde, C. Marín, y P.M. Madriz. 2007. Comparación de tres métodos para estimar estabilidad del rendimiento en nueve variedades de algodón. Interciencia 32(5):344-348.

Greveniotis, V., and E. Sioki. 2017. Genotype by environment interactions on cotton fiber traits and their implications on variety recommendation. J. Agric. Stud. 5(2):86-106. doi:10.5296/jas.v5i2.10762

Liu, S.M., G.A. Constable, P.E. Reid, W.N. Stiller, and B.R. Cullis. 2013. The interaction between breeding and crop management in improved cotton yield. F. Crop Res. 148:49-60. doi:10.1016/j.fcr.2013.04.006

Lozano-Ramírez, Á., A. Santacruz-Varela, F. San-Vicente-García, J. Crossa, J. Burgueño, y J.D. Molina-Galán. 2015. Modelación de la interacción genotipo x ambiente en rendimiento de híbridos de maíz blanco en ambientes múltiples. Rev. Fitotec. Mex. 38:337-347.

Martínez-Reina, A.M., y M.J. Hernández. 2015. La competitividad del algodón colombiano frente a los principales países productores mediante el enfoque de costos de producción. Corpoica Cienc. Tecnol. Agropecu. 16:189-215. 
Mehboob-ur-Rahman, T. Shaheen, N. Tabbasam, M. Atif-Iqbal, M. Ashraf, Y. Zafar, and A.H. Paterson. 2012. Cotton genetic resources. A review. Agron. Sustain. Dev. 32:419-432. doi:10.1007/s13593-011-0051-Z

Mudada, N., J. Chitamba, T.O. Macheke, and P. Manjeru. 2017. Genotype $\times$ environmental interaction on seed cotton yield and yield components. OALib 4(11):1-22. doi:10.4236/oalib.1103192

OECD (Organisation for Economic Co-operation and Development), and FAO. 2018. Cotton. In: OECD, and FAO, editors, OECD-FAO agricultural outlook 2018-2027. Chapter 10. OECD Publishing, Paris, FRA. p. 207-215.

Oliveira, E.J. de, J.P.X. de Freitas, and ON de Jesus. 2014. AMMI analysis of the adaptability and yield stability of yellow passion fruit varieties. Sci. Agric. 71(2):139-145. doi:10.1590/S0103-90162014000200008

Orawu, M., G. Amoding, L. Serunjogi, G. Ogwang, and C. Ogwang. 2017. Yield stability of cotton genotypes at three diverse agro-ecologies of Uganda. J. Plant Breed Genet. 5(3):101-114.

Palomo-Gil, A., A. Gaytán-Mascorro, R. Faz-Contreras, D.G. Reta-Sánchez, and E. Gutiérrez-Del Río. 2004. Cotton yield and fiber quality in response to nitrogen rate and number of irrigations. Terra Latinoam. 22:299-305.

R Core Team. 2015. R: A language and environment for statistical computing. R Project. http://www.r-project.org/ (accessed Apr. 2019).

Rodríguez-Pérez, J.E., J. Sahagún-Castellanos, H. Villaseñor-Mir; J.D. Molina-Galán, A. Martínez-Garza. 2005. La interacción genotipo x ambiente en la caracterización de áreas temporales de producción de trigo. Agrociencia 39(1):51-64.

Sareen, S., B. Singh Tyagi, A. Kumar Sarial, V. Tiwari, and I. Sharma. 2014. Trait analysis, diversity, and genotype $\times$ environment interaction in some wheat landraces evaluated under drought and heat stress conditions. Chil. J. Agric. Res. 74(2):135-142. doi:10.4067/S0718-58392014000200002

Vargas, A.E., D. Baena, and J.E. Vargas. 2016. Analysis of stability and adaptability of QPM hybrids of maize growing in different Colombian agroecological zones. Acta Agron. 65:72-79. doi:10.15446/acag.v65n1.43417

Wendel, J.F., C.L. Brubaker, and T. Seelanan. 2010. The origin and evolution of gossypium. In: J.M. Stewart et al., editors, Physiology of cotton. Springer, Dordrecht, NLD. p. 1-18. doi:10.1007/978-90-481-3195-2_1

Williams, H., V. Pecina, F. Zavala, N. Montes, A.J. Gámez, G. Arcos, M.A. García, S. Montes, and L. Alcalá. 2010. Finlay and Wilkinson model vs. AMMI model in the analysis of genotype-environment interaction in sorghum. Rev. Fitotec. Mex. $33: 117-123$.

Zhi, X., Y. Han, Y. Li, G. Wang, W. Du, X. Li, S. Mao, and L. Feng. 2016. Effects of plant density on cotton yield components and quality. J. Integr. Agric. 15:1469-1479. doi:10.1016/S2095-3119(15)61174-1 